\title{
ONSET OF ITINERANT FERROMAGNETISM IN COBALT-NICKEL HEUSLER-TYPE COMPOUNDS
}

\author{
J. PIERRE ${ }^{a}$, R.V. Skolozdra ${ }^{a, b}$ AND YU.K. GoRelenko ${ }^{b}$ \\ ${ }^{a}$ Laboratoire L. Néel, CNRS, 166X, 38042 Grenoble, France \\ ${ }^{b}$ Department of Inorganic Chemistry, I. Franko University, 290005 Lviv, Ukraine
}

The magnetic properties of $\mathrm{Co}-\mathrm{Ni}$ compounds with $\mathrm{MnCu}_{2} \mathrm{Al}$ or MgAgAs-type structure (the Heusler phases) were studied. Paramagnetic, ferromagnetic moments, Curie's temperature increase with Co content. The ratio of ordered to paramagnetic moment varies with the Curie temperature following itinerant ferromagnetism models. A metal-insulator transition occurs in the $\mathrm{TiCo}_{x} \mathrm{Ni}_{1-x} \mathrm{Sn}$ series, which causes a more rapid drop of the Curie temperature than in $\mathrm{TiCo}_{x} \mathrm{Ni}_{2-x} \mathrm{Sn}$ solid solutions which remain metallic.

PACS numbers: 75.20.En, 75.10.Lp, 75.50.Cc

\section{Introduction}

The Heusler phases are famous ternary intermetallics, of the type $\mathrm{MM}_{2}^{\prime} \mathrm{X}$, where $\mathrm{M}$ and $\mathrm{M}^{\prime}$ are transition metals. $\mathrm{Mn}, \mathrm{Fe}$ and Co compounds are often ferromagnets, whereas Ni compounds are the Pauli paramagnets [1]; beyond them, Mn compounds exhibit a quasi-localized magnetism [2] due to the half-filled $3 d$ band, but Co compounds are examples of itinerant magnetism.

We studied the magnetic properties of three series of Co and Ni Heusler phases with the general formula $\mathrm{TiCo}_{x} \mathrm{Ni}_{y} \mathbf{\Sigma}_{2-x-y} \mathrm{Sn}$, where $\mathrm{m}$ is a vacancy in the lattice. $\mathrm{TiCo}_{2} \mathrm{Sn}$ [1], TiCoSn [3] are metallic ferromagnets, $\mathrm{TiNi}_{2} \mathrm{Sn}$ a metallic Pauli paramagnet, and TiNiSn a nonmagnetic semiconductor with a small gap [4]. Among these compounds, we selected three series of solid solutions: $\mathrm{TiCo}_{x} \mathrm{Ni}_{2-x} \mathrm{Sn}$, $\mathrm{TiCo}_{x} \mathbf{m}_{2-x} \mathrm{Sn}$ and $\mathrm{TiCo}_{x} \mathrm{Ni}_{1-x} \mathrm{Sn}$. In the first series, there is a progressive filling of the $3 d$ band with increasing $\mathrm{Ni}$ content whereas a simple dilution of Co by vacancies occurs in the second. The third series undergoes a metal-nonmetal transition.

\section{Preparation and crystallography}

Samples were prepared using an arc furnace, and were annealed during one month at $650^{\circ} \mathrm{C}$ or during one week at $800^{\circ} \mathrm{C}$, with no significant changes in their properties. A single cubic phase is observed, the lattice parameter changes slightly with the composition and the values for the extremes are: $\mathrm{TiCo}_{2} \mathrm{Sn}-$ 
6.067 $\AA, \mathrm{TiNi}_{2} \mathrm{Sn}-6.097 \AA, \mathrm{TiCoSn}-5.997 \AA, \mathrm{TiNiSn}-5.921 \AA . \mathrm{TiCoSn}$ and TiNiSn crystallize with the MgAgAs structure, which has a vacant site. However, careful investigations reveal that small percentages of $\mathrm{Sn}$ and Co exist on the "vacant site" in TiCoSn. Neutron diffraction with a high resolution diffractometer shows that there is a spread in lattice parameter for the compound $\mathrm{TiCo}_{1.6} \mathrm{Sn}$, thus inhomogeneities occur in the distribution of vacancies, with a possible consequence on the spread of the Curie points.

\section{Physical properties}

Magnetic measurements were performed in fields up to 7 teslas between $1.5 \mathrm{~K}$ and $500 \mathrm{~K}$. Transport properties were measured below room temperature. We followed the variations of the ferromagnetic Curie point $T_{\mathrm{C}}$, ordered magnetic moment $M(T)$, as well as the Curie-Weiss parameter $T_{\mathrm{p}}$ and effective moment $M_{\text {eff }}$ when a Curie-Weiss behaviour occurs at high temperatures. In the following, $M$ is the moment per formula unit, whereas $\mu$ is the moment per Co atom (assuming it is the only magnetic species). We assume the local moment approximation to deduce the effective moment.

In the three series, a Curie-Weiss behaviour is observed for large enough Co concentrations. $T_{\mathrm{C}}, M(0), T_{\mathrm{p}}$ and $M_{\text {eff }}$ decrease with decreasing Co content; the ratio $M(0) / M_{\text {eff }}$ also decreases similarly to the decrease in the Curie temperature. This is a classical result of itinerant magnetism pointed out by Rhodes and Wohlfarth [5] which is related to the amplitude of the band splitting proportional to intersite interactions.

In the series $\left(\mathrm{Co}_{x} \mathrm{Ni}_{2-x}\right)$, the limit of ferromagnetism is observed to be $x_{\mathrm{Co}}=1.2 . T_{\mathrm{C}}$ and $T_{\mathrm{p}}$ decrease almost linearly with $x, T_{\mathrm{p}}$ being smaller than $T_{\mathrm{C}}$. The difference between them is largest for low Co concentrations, pointing to large spin fluctuations in the vicinity of the ordering point. The susceptibility for Ni-rich compounds turns progressively to constant paramagnetism.

In the $\left(\mathrm{Co}_{x} \mathbf{m}_{2-x}\right)$ series, $T_{\mathrm{C}}$ and $T_{\mathrm{p}}$ decrease less rapidly with $x$ than in the first series, indicating that Co dilution is less efficient in decreasing the local moment on Co than adding electrons in the band. Meanwhile, the ferromagnetic Curie point and ordered moment decrease more rapidly than the paramagnetic temperature and effective moment respectively, following the reduction of intersite interactions.

Another remarkable feature in the $\left(\mathrm{Co}_{x} \omega_{2-x}\right)$ series is the non-linear variation of saturation and effective moments with the Co content. A rapid increase occurs for $\mu(0), \mu_{\text {eff }}$ and $T_{\mathrm{C}}$ between $x=1.6$ and 1.9. This may be due either to a rapid change in the density of states at the Fermi level, or to the occurrence of two different sites in these compounds: the partly vacant site would bear a smaller magnetic moment than the full one; it may even not fulfil the Stoner criterion for low concentrations and experience induced magnetism.

The properties of these two series are described elsewhere in more details [6].

For the $\left(\mathrm{Co}_{x} \mathrm{Ni}_{1-x}\right)$ series, the experimental value of the resistivity at $4 \mathrm{~K}$ increases from $25 \times 10^{-6} \mathrm{Ohm} \cdot \mathrm{cm}$ for TiCoSn to $25 \times 10^{-3} \mathrm{Ohm} \cdot \mathrm{cm}$ for TiNiSn, that is by three orders of magnitude. For TiNiSn, we have to deal with a heavily 
doped or degenerate semiconductor. From the Hall effect, between 100 and $260 \mathrm{~K}$, majority carriers are electrons. A plot of $\log (R)$ versus $1 / T$ gives a minimum value of the energy gap of $450 \mathrm{~K}$ for TiNiSn, and $80 \mathrm{~K}$ for $\mathrm{TiCo}_{0.2} \mathrm{Ni}_{0.8} \mathrm{Sn}$, which may not represent the complete energy gap, but the distance between the valence (or conduction) band and impurity levels.

Ferromagnetism occurs for Co concentrations larger than 0.45 , which is also the limit between metallic and semiconducting behaviour. At variance with the previous series, the magnetism changes from itinerant in TiCoSn to localised for Co diluted in TiNiSn. Indeed, a Curie-Weiss behaviour may be observed for small Co concentrations at low temperatures, where the susceptibility may be written as $X=X_{0}+C_{M} / T$, where $X_{0}$ is temperature independent. Saturation and effective moments per formula are given in Fig. 1. The Curie constant per Co ion is $C_{M} / x$,

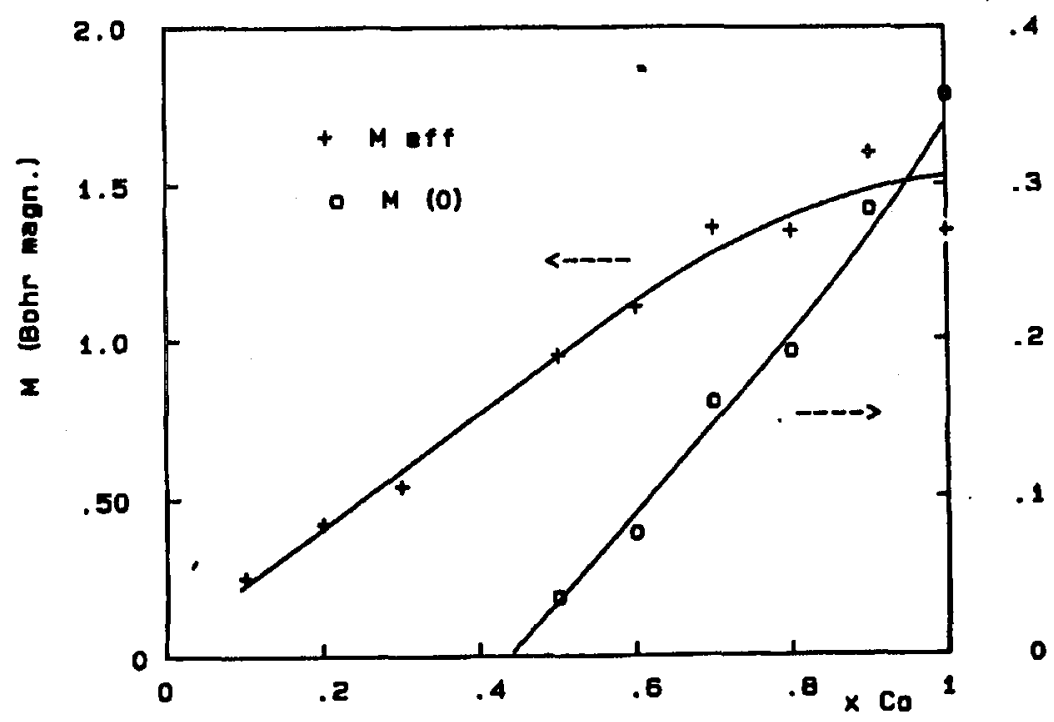

Fig. 1. Effective moment and saturation magnetization (per formula) in $\mathrm{TiCo}_{x} \mathrm{Ni}_{1-x} \mathrm{Sn}$ series, versus Co content.

hence we obtain the effective magnetic moment of Co. Surprisingly, this moment slightly changes between $\mathrm{TiCoSn}\left(1.35 \mu_{\mathrm{B}}\right)$ to $\mathrm{TiCo}_{0.1} \mathrm{Ni}_{0.9} \mathrm{Sn}\left(1.1 \mu_{\mathrm{B}}\right)$, thus Co diluted in TiNiSn keeps a local moment similar to its value for itinerant TiCoSn.

On the contrary, intersite interactions are strongly reduced by the decrease in the density of states. A good manner to visualize this feature is to plot $T_{\mathrm{C}}$ versus the saturation moment $M(0)$ : for the two first series, $T_{\mathrm{C}}$ varies nearly linearly with $M$, whereas it varies as $M^{1.7}$ in $\left(\mathrm{Co}_{x} \mathrm{Ni}_{1-x} \omega\right)$ series $\left(M^{2}\right.$ in the theory of weak ferromagnetism). Thus intersite interactions drop rapidly to zero in this last series, while the local effective moment on Co keeps a nearly constant value as the metal-nonmetal transition occurs.Conversely, $T_{\mathrm{C}}$ follows an unique law as function 


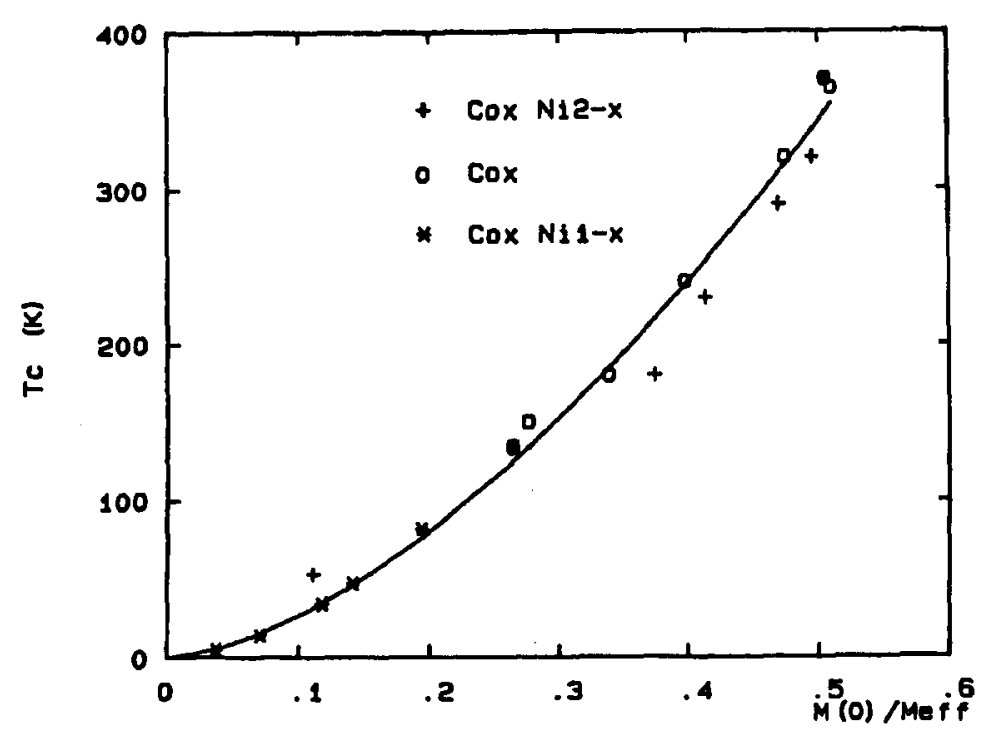

Fig. 2. Ferromagnetic Curie temperature versus the ratio $M(0) / M_{\text {eff }}$ in $\mathrm{TiCo}_{x} \mathrm{Ni}_{1-x} \mathrm{Sn}$ series.

TABLE

Magnetic properties for some selected ferromagnetic compounds.

Moments are given per cobalt atom.

\begin{tabular}{l|c|c|c|c|c}
\hline \multicolumn{1}{c|}{ Compound } & $T_{\mathrm{p}}[\mathrm{K}]$ & $\mu_{\mathrm{eff}}\left[\mu_{\mathrm{B}}\right]$ & $\mu(0)\left[\mu_{\mathrm{B}}\right]$ & $T_{\mathrm{C}}[\mathrm{K}]$ & $\mu_{\mathrm{eff}} / \mu(0)$ \\
\hline $\mathrm{TiCo}_{2} \mathrm{Sn}$ & 370 & 1.96 & 0.98 & 370 & 1.98 \\
$\mathrm{TiCoSn}$ & 158 & 1.35 & 0.357 & 134 & 3.78 \\
$\mathrm{TiCo}_{1.4} \mathrm{Ni}_{0.6} \mathrm{Sn}$ & 115 & 1.42 & 0.166 & 53 & 8.6 \\
$\mathrm{TiCo}_{0.5} \mathrm{Ni}_{0.5} \mathrm{Sn}$ & 7.5 & 1.32 & 0.074 & 5 & 18
\end{tabular}

of $M(0) / M_{\text {eff }}$ for the 3 series (Fig. 2), which expresses the Rhodes-Wohlfarth law [5].

Resistivity and magnetoresistance were measured for some compounds. Among metallic systems, a negative temperature coefficient is observed at low temperatures for alloys in the vicinity of the magnetic-nonmagnetic limit $\left(\mathrm{TiCo}_{1.2} \mathrm{Ni}_{0.8} \mathrm{Sn}, \mathrm{TiCo}_{0.5} \mathrm{Ni}_{0.5} \mathrm{Sn}\right.$ ), which may be due to spin fluctuations. The first compound, which has $T_{\mathrm{p}}=+71 \mathrm{~K}$ and does not order, is a $3 d$ counterpart of heavy fermion systems. A high positive value of the magnetoresistance is observed in TiCoSn at $4 \mathrm{~K}$. This may be related to half-metallic conduction, a phenomenon which occurs in isomorphous NiMnSb [7], and arises from a strong difference between spin-up and spin-down subbands in the ferromagnetic state. 


\section{Conclusion}

The evolution of magnetic properties with Co concentration shows the classical features of itinerant magnetism in these compounds. The study of three series of solutions allowed to separate the influence of pure magnetic dilution, filling of the $3 d$ band by an extra electron and that of the metal-semiconductor transition, on the building of the local moment of the Co site and on the interactions between sites.

\section{Acknowlegdments}

We thank A.M. Kouacou (Labo L. Néel) who performed some of the magnetic measurements, and J. Rodriguez (Labo L. Brillouin, Saclay) for his help during neutron diffraction experiments.

\section{References}

[1] K.R.A. Ziebeck, P.J. Webster, J. Phys. Chem. Solids 35, 1 (1974).

[2] J. Kübler, A.R. Williams, C.B. Sommers, Phys. Rev. B 28, 1745 (1983).

[3] R.V. Skolozdra, Yu.V.Stadnyk, Yu.K. Gorelenko, E.E. Terletskaya, Sov. Phys. Solid State 32, 1536 (1990).

[4] F.G. Aliev, V.V. Kozyrkov, V.V. Moschalkov, R.V. Skolozdra, K. Durczewski, Z. Phys. B 80, 353 (1990).

[5] P. Rhodes, W.P. Wohlfarth, Proc. R. Soc. 273, 247 (1963).

[6] J. Pierre, R.V. Skolozdra, Yu.V.Stadnyk, J. Magn. Magn. Mater., in press.

[7] R.A. de Groot, F.M. Mueller, P.G. van Engen, K.H.J. Buschow, Phys. Rev. Lett. 50, 2024 (1983). 